\title{
On Collision Avoiding Fixed-Time Flocking with Measurable Diameter to a Cucker-Smale-Type Self-Propelled Particle Model
}

\author{
Qizhen Xiao, Hongliang Liu (iD, Zhenghua Xu iD, and Zigen Ouyang \\ School of Mathematics and Physics, University of South China, Hengyang 421001, China \\ Correspondence should be addressed to Hongliang Liu; math_lhliang@163.com and Zhenghua Xu; 476787291@qq.com
}

Received 6 February 2020; Accepted 26 March 2020; Published 5 May 2020

Academic Editor: Qingling Wang

Copyright (C) 2020 Qizhen Xiao et al. This is an open access article distributed under the Creative Commons Attribution License, which permits unrestricted use, distribution, and reproduction in any medium, provided the original work is properly cited.

\begin{abstract}
Dealing with the fixed-time flocking issue is one of the most challenging problems for a Cucker-Smale-type self-propelled particle model. In this article, the fixed-time flocking is established by employing a fixed-time stability theorem when the communication weight function has a positive infimum. Compared with the initial condition-based finite-time stability, an upper bound of the settling time in this paper is merely dependent on the design parameters. Moreover, the size of the final flocking can be estimated by the number of particles and the initial states of the system. In addition, a sufficient condition is formulated to guarantee that all particles do not collide during the process of the flocking. These results can give a reasonable explanation to some flocking phenomena such as bird flocks, fish schools, or human group behaviors. Finally, three numerical examples are granted to display the performance of the obtained results.
\end{abstract}

\section{Introduction}

In the last few years, the cooperative control of self-propelled agent systems has attracted considerable attention, mainly due to its broad range of potential applications including formation control [1], attitude alignment [2], and target tracking [3]. Such coordinative motion is typically inspired by the emergent collective behaviors observed in nature. Flocking, as an important emergent collective behavior, has been studied with great attention. Readers are referred to [4-15] and references therein for more details.

Let us point out that most aforementioned works mainly addressed the asymptotical behaviors, which mean that the flocking can only occur as time approaches to infinity. However, in our real life, phenomena in which particles reach to flock in a short time are ubiquitously observed. Take bird flocks, for example, individuals can form a new flock after adjusting their states in a short time when they are disturbed by the external environment. Although we do not know deeply about the traits intrinsic that why birds can occur as flocks in a very short time, this common phenomenon has inspired us to focus on the finite-time control problems. In 1986, Haimo firstly investigated the finite-time control problem in the pioneer work [16]. Based on the results of the benchmark works of Bhat and Bernstein [17], the issue of the finite-time cooperative control for multiagent systems has developed dramatically. Accordingly, there is a large body of the literature on the research in this field, which is impossible to cite here. Instead, we refer to several recent works as representatives. For multiagent systems with unknown inherent nonlinear dynamics, Cao and Ren [18] demonstrated that the consensus can be achieved in a finite-time by using graph theory and a finitetime theorem. For multiagent systems with discontinuous inherent dynamics, Ning et al. [19] considered the finitetime and fixed-time leader-following consensus by combining graph theory and discontinuous analysis. For discontinuous complex networks, Ji et al. [20] studied the finitetime and fixed-time synchronization by establishing a new finite-time and fixed-time theorems. For another relative interesting results on finite-time problems, we refer to [21-31] and therein references. As far as we observe there are a lot of works on the finite-time consensus problem for multiagent systems, but few results on finite-time flocking for multiagent systems available in the literature [32, 33]. More precisely, the results of [32] show the finite-time 
flocking of a Cucker-Smale-type self-propelled particle model can be achieved when the communication weight function has a positive infimum. In [33], the results demonstrate that the finite-time flocking occurs when the communication weight function under long-range interactions.

It is worth pointing out that the estimation of settling time function for finite-time stability is heavily depending on the initial states of systems [16]. Therefore, from a practical point of view, this restrict limits especially the practical applications, since the initial states of the systems are unavailable in advance. To overcome this shortcoming, an extension of the finite-time stability and fixed-time stability is discovered $[34,35]$. The fixed-time stability exhibits an elegant property in some applications. We refer to two review works for an overview by $[36,37]$ and some interesting results in fixed-time consensus [19, 27, 29, 37], fixed-time synchronization [38], and fixed-time bipartite flocking [39]. Note however, there are no papers addressing the fixed-time flocking with collision avoidance and the final size of the group, which will be the subject of this paper. Naturally, in the present paper, we will consider the following problems:

(1) Under what conditions can the flocking emerge in a fixed time?

(2) If the flocking occurs in a fixed-time, can the diameter of the final group be estimated?

(3) Under what conditions could particles be ensured not to collide with each other?

To solve the abovementioned problems (1)-(3), we first propose a continuous non-Lipschitz type model (since the finite-time flocking cannot occur in the Lipschitz model $[16,32,33])$ and then carry out the fixed-time stability analysis which shows that if the communication weight function satisfies a lower bound condition then flocking can occur within a fixed-time. Consequently, the novelty of the present paper lies in the following aspects. Firstly, the right term of our model does not satisfy the Lipschitz condition, which is different from the classical Cucker-Smale model $[18,40,41]$, where the flocking only occurs asymptotically. In addition, it is not necessary to require that the communication weight function is a nonincreasing function but only has a positive infimum. By using a fixed-time stability theorem, the fixed-time flocking result is obtained in this present paper. Secondly, unlike the results in $[32,33]$, in which flocking is achieved in a finite-time which is depending on the initial states of network agents, we investigate the fixed-time flocking and estimate the upper bound of the settling time which is merely depending on parameters. This provides additional options for designers in practical scenarios where initial conditions are unavailable. Thirdly, the results in $[32,33]$ show that the particles may collide in the process of flocking. However, a new sufficient condition is presented in this article to guarantee that particles do not collide during the process of flocking. Finally, the largest diameter of final flocking group can be estimated by the initial states and the parameters.
The rest of this paper is organized as follows. In Section 2 , some necessary lemmas and a fixed-time stability theorem are presented, which are the main tools to study the fixedtime flocking problem. In Section 3, a fixed-time flocking with collision avoidance theorem for the Cucker-Smale-type self-propelled particle model is established. Meanwhile, the size of the final flocking can be estimated by the initial data and parameters in this section. Three illustrative simulated examples are presented to demonstrate the theorem analysis in Section 4. Finally, a brief conclusion is presented in Section 5 .

\section{Preliminaries and Problem Formulation}

2.1. Preliminaries. Consider the following dynamical system:

$$
\dot{x}(t)=g(t, x(t)), \quad x\left(t_{0}\right)=x_{0},
$$

where $x \in \mathbb{R}^{d}, g: \mathbb{R}^{+} \times \mathbb{R}^{d} \longrightarrow \mathbb{R}^{d}$ is a nonlinear function which may be discontinuous, then the solutions of (1) are understood in the sense of Filippov and Arscott [42]. Assume that the origin is an equilibrium point of (1). Hereafter, without loss of generality, it is assumed $t_{0}=0$.

Definition 1 (see [35]). The origin of (1) is said to be globally finite-time stable if it is globally asymptotically stable and any solution $x\left(t, x_{0}\right)$ of (1) reaches the equilibrium at some finite moment, i.e.,

$$
x\left(t, x_{0}\right) \equiv 0, \quad \forall t \geq T\left(x_{0}\right),
$$

where $T: \mathbb{R}^{d} \longrightarrow \mathbb{R}_{+} \cup\{0\}$ is the setting-time function.

Definition 2 (see [35]). The origin of (1) is said to be globally fixed-time stable if it is globally finite-time stable and the settling time function $T\left(x_{0}\right)$ is bounded, namely, there exists $T_{\max }>0$ such that $T\left(x_{0}\right) \leq T_{\max }$ for all $x_{0} \in \mathbb{R}^{d}$.

Lemma 1 (see [43])

(i) If $a_{i}>0, i=1, \ldots, N$, and $p>r>0$, then

$$
N^{(1 / p)-(1 / r)}\left(\sum_{i=1}^{N} a_{i}^{r}\right)^{(1 / r)} \leq\left(\sum_{i=1}^{N} a_{i}^{p}\right)^{(1 / p)} \leq\left(\sum_{i=1}^{N} a_{i}^{r}\right)^{(1 / r)} .
$$

(ii) If $a_{1}, a_{2}, \ldots, a_{n} \geq 0$ and $0<p \leq 1$, then the following inequality holds:

$$
\left(\sum_{i=1}^{n} a_{i}\right)^{p} \leq \sum_{i=1}^{n} a_{i}^{p}
$$

Lemma 2 (see [27]). If $a_{1}, a_{2}, \ldots, a_{n} \geq 0$ and $p>1$, then the following inequality holds: 


$$
n^{1-p}\left(\sum_{i=1}^{n} a_{i}\right)^{p} \leq \sum_{i=1}^{n} a_{i}^{p}
$$

Lemma 3 (see [38]). If there exists a regular, positive, definite, and radially unbounded function $V(x): \mathbb{R}^{n} \longrightarrow \mathbb{R}$ such that any solution $x(t)$ of (1) satisfies the inequality

$$
\frac{\mathrm{d}}{\mathrm{d} t} V(x(t)) \leq-a V^{\delta}(x(t))-b V^{\theta}(x(t)), \quad x(t) \in \mathbb{R}^{n} \backslash\{0\},
$$

where $a>0, b>0, \delta>1$, and $0 \leq \theta<1$, then the origin of system (1) is fixed-time stable, and the settling time function $T$ is estimated by

$$
T \leq T_{\max }:=\frac{1}{b}\left(\frac{b}{a}\right)^{((1-\theta) /(\delta-\theta))}\left(\frac{1}{1-\theta}+\frac{1}{\delta-1}\right) .
$$

2.2. Problem Formulation. To research the flocking of birds, Cucker and Smale [44] in 2007 introduced the following model (short for C-S model):

$$
\left\{\begin{array}{l}
\dot{x}_{i}=v_{i}, \quad i=1, \ldots, N, \quad t>0 \\
\dot{v}_{i}=\frac{1}{N} \sum_{j=1, j \neq i}^{N} \psi\left(\left\|x_{i}-x_{j}\right\|\right)\left(v_{j}-v_{i}\right),
\end{array}\right.
$$

where $x_{i}$ and $v_{i}$ denote the position and velocity of the $i$ th particle, and the communication rate $\psi$ is assumed to be satisfied:

$$
\psi(s)=\frac{K}{\left(1+s^{2}\right)^{\beta}}, \quad K>0, \beta \geq 0 .
$$

The results in [44] show that the global unconditional flocking occurs as $t \longrightarrow \infty$ for $\beta<(1 / 2)$, while the conditional flocking appears under some restricted conditions on the initial positions and velocities as $t \longrightarrow \infty$ for $\beta<(1 / 2)$. Motivated by the C-S model, in order to study the finite-time flocking of the self-propelled particle model, Han et al. [32] purposed the following Cucker-Smale-type self-propelled particle model:

$$
\left\{\begin{array}{l}
\dot{x}_{i}=v_{i}, \quad i=1, \ldots, N, \quad t>0, \\
\dot{v}_{i}=\sum_{j=1, j \neq i}^{N} \psi\left(\left\|x_{i}-x_{j}\right\|\right) \operatorname{sig}\left(v_{j}-v_{i}\right)^{\theta},
\end{array}\right.
$$

where $\psi$ denotes the communication weight function between agents $i$ and $j$ satisfying

$$
\inf _{s \geq 0} \psi(s) \geq \psi^{*}>0 \text {. }
$$

Hereinafter, $\|\cdot\|$ denotes the $l_{2}$-norm and

$$
\operatorname{sig}\left(v_{j}-v_{i}\right)^{\theta}=\left\{\operatorname{sgn}\left(v_{j 1}-v_{i 1}\right)\left|v_{j 1}-v_{i 1}\right|^{\theta}, \ldots, \operatorname{sgn}\left(v_{j d}-v_{i d}\right)\left|v_{j d}-v_{i d}\right|^{\theta}\right\}^{T},
$$

with $\theta \in(0,+\infty)$ and $\operatorname{sgn}(\cdot): \mathbb{R} \mapsto\{-1,0,1\}$ as the signum function. By using a finite-time stability theorem, the authors [32] proved that, for $0<\theta<1$, the flocking can occur in a finite-time (the upper bound of it is heavily depends on the initial data of the systems). Unfortunately, the author did not indicate that whether the particles collide or not during the process of the flocking.

Motivated by [32], the main goal of this paper is to investigate the noncollision fixed-time flocking and estimate the size of it. To this end, according to the results of $[16,32,33]$ that the finite-time stability cannot take place in Lipschitz continuous system, we consider an interacting particle system consisting of $N$ identical autonomous particles with a new non-Lipschitz type given by the following system:

$$
\left\{\begin{array}{l}
\dot{x}_{i}=v_{i}, \quad i=1, \ldots, N, \quad t>0, \\
\dot{v}_{i}=\frac{1}{N} \sum_{j=1, j \neq i}^{N} \psi\left(\left\|x_{i}-x_{j}\right\|\right)\left(\sum_{\kappa\{-1,1\}} K_{\kappa} \operatorname{sig}\left(v_{j}-v_{i}\right)^{1+2 \kappa \alpha}\right),
\end{array}\right.
$$

subject to the initial data

$$
\left(x_{i}, v_{i}\right)(0)=:\left(x_{i 0}, v_{i 0}\right), \quad i=1, \ldots, N,
$$

where $0<\alpha<(1 / 2), x_{i}=\left(x_{i 1}, x_{i 2}, \ldots, x_{i}{ }_{d}\right) \in \mathbb{R}^{d}$ and $v_{i}=$ $\left(v_{i 1}, v_{i 2}, \ldots, v_{i}{ }_{d}\right) \in \mathbb{R}^{d}$ denote the position and velocity of $i$ th particle at time $t$, respectively, $N$ is the number of particles, while $K_{\kappa}(\kappa=-1,1)$ are positive constants measuring the coupling strength and the functions $\psi$ and sig are defined in (11) and (12), respectively.

For vectors $x_{i}, v_{i} \in \mathbb{R}^{d}$, the Euclidean norm and the inner product are given by

$$
\begin{aligned}
\left\|x_{i}\right\| & :=\left(\sum_{k=1}^{d}\left|x_{i k}\right|^{2}\right)^{(1 / 2)}, \\
\left\langle x_{i}, v_{i}\right\rangle & :=\sum_{k=1}^{d} x_{i k} v_{i k},
\end{aligned}
$$

where $x_{i k}$ and $v_{i k}$ are the $i$ th components of $x_{i}$ and $v_{i}$, respectively. Similarly to $[32,45]$, we set the center of mass system $(\bar{x}, \bar{v})$ as follows: 


$$
\begin{gathered}
\bar{x}:=\frac{1}{N} \sum_{i=1}^{N} x_{i}, \\
\bar{v}:=\frac{1}{N} \sum_{i=1}^{N} v_{i} .
\end{gathered}
$$

It follows from the definition of $\operatorname{sig}(\cdot)$ that

$$
\operatorname{sig}\left(v_{j}-v_{i}\right)^{\theta}=-\operatorname{sig}\left(v_{i}-v_{j}\right)^{\theta}, \quad \forall \theta>0 .
$$

Furthermore,

$$
\sum_{i, j=1}^{N} \psi\left(\left\|x_{i}-x_{j}\right\|\right) \operatorname{sig}\left(v_{j}-v_{i}\right)^{\theta}=0, \quad \forall \theta>0 .
$$

Thus, from (13), we obtain

$$
\begin{aligned}
& \frac{\mathrm{d} \bar{x}}{\mathrm{~d} t}=\bar{v}, \\
& \frac{\mathrm{d} \bar{v}}{\mathrm{~d} t}=0,
\end{aligned}
$$

which admit the explicit solution $\bar{x}(t)=\bar{x}(0)+\bar{v}(0) t$ and $\bar{v}(t)=\bar{v}(0)$ for $t \geq 0$. Hence, we may assume, without loss of generality that the center of mass coordinate of the system in phase space at time $t$ is fixed at zero, i.e.,

$$
\begin{aligned}
& \bar{x}(t)=0, \\
& \bar{v}(t)=0,
\end{aligned}
$$

which implies that

$$
\begin{aligned}
& \sum_{i=1}^{N} x_{i}(t)=0 \\
& \sum_{i=1}^{N} v_{i}(t)=0 \\
& t \geq 0
\end{aligned}
$$

Let $\left(\hat{x}_{i}, \widehat{v}_{i}\right):=\left(x_{i}-\bar{x}, v_{i}-\bar{v}\right)$ be the fluctuations around the center of the mass system. It is easy to see that $\hat{x}_{c}=$ $1 / N \sum_{i=1}^{N} \widehat{x}_{i}=0$ and $\widehat{v}_{c}=1 / N \sum_{i=1}^{N} \widehat{v}_{i}=0$. Then, system (13) can be written as

$$
\left\{\begin{array}{l}
\dot{\hat{x}}_{i}=\widehat{v}_{i}, \quad i=1, \ldots, N, t>0, \\
\dot{\hat{v}}_{i}=\frac{1}{N} \sum_{j=1, j \neq i}^{N} \psi\left(\left\|\widehat{x}_{i}-\widehat{x}_{j}\right\|\right)\left(\sum_{\kappa\{-1,1\}} K_{\kappa} \operatorname{sig}\left(\widehat{v}_{j}-\widehat{v}_{i}\right)^{1+2 \kappa \alpha}\right),
\end{array}\right.
$$

which means that the new variable $\left(\widehat{x}_{i}, \widehat{v}_{i}\right)$ satisfies equation $(13)$ and $\left(\widehat{x}_{c}, \widehat{v}_{c}\right) \equiv(0,0)$. For simplicity, we drop the hat notion in the microscopic variables and replace $\left(\hat{x}_{i}, \widehat{v}_{i}\right)$ with $\left(x_{i}, v_{i}\right)$ in the present paper. That is, the dynamics behaviors of system (22) equate to the dynamics behaviors of system (13).

Remark 1. The right-hand side of the second equation (i.e., controller) in (13) or (22) is consisting of two different terms since considering two scenarios in the system. That is, for $0<\alpha<1 / 2$, if the difference in velocity between any two particles is smaller than one unit, the term $K_{-1} \operatorname{sig}\left(\widehat{v}_{j}-\widehat{v}_{i}\right)^{1-2 \alpha}$ (i.e., $\kappa=-1$ ) plays a leading role in promoting the speed to converge. Conversely, if the difference in velocity between any two particles is larger than one unit, then the term $K_{1} \operatorname{sig}\left(\widehat{v}_{j}-\widehat{v}_{i}\right)^{1+2 \alpha}$ (i.e., $\kappa=1$ ) plays the dominant role in driving the speed to converge. Unlike system (7), this controller can guarantee the flocking occurs in a fixed time, which can be estimated merely by the parameters.

Remark 2. In our model (13) or (22), the communication weight function $\psi$ defined in (11) means that all the particles in the systems exchange information with each other. In this sense, the communication function $\psi$ is well defined in (11). However, different from (6), it cannot adequately characterize the relationship between the intensity of information and the distance between particles.

\section{Main Results}

The objective in this section is to study the fixed-time flocking of system (13). Motivated by [4, 11, 25, 45], the particles in this paper are viewed as the points without physical sizes as well. Now, we present the definition of noncollision fixed-time flocking as follows:

Definition 3. We say that system (13) has a fixed-time flocking if there exist a time $T \geq 0$ and a constant $C>0$ such that

$$
\begin{aligned}
\lim _{t \longrightarrow T}\left\|v_{i}(t)-v_{j}(t)\right\| & =0, \\
\left\|v_{i}(t)-v_{j}(t)\right\| & =0, \\
\operatorname{up}_{0 \leq t<\infty}\left\|x_{i}(t)-x_{j}(t)\right\| & \leq C, \\
\forall t & \geq T,
\end{aligned}
$$

where $T$ is called the settling time and the upper bound of it can be estimated as a fixed $T_{\max }$ that is independent of initial states of system (13). Furthermore, if system (13) has a fixedtime flocking and

$$
\min _{i \neq j}\left\|x_{i}(t)-x_{j}(t)\right\|>0, \quad t \geq 0
$$

then we say that system (13) has a noncollision fixed-time flocking.

Now, we state the main results of this section as follows.

Theorem 1. For $0<\alpha<(1 / 2)$, if $K_{\kappa}>0, \kappa=\{-1,1\}$, and (11) holds, then system (13) has a fixed-time flocking. Moreover, the settling time can be estimated by 


$$
T \leq T_{\max }=\frac{2 N}{\alpha \psi^{*} K_{-1}(2 N)^{1-2 \alpha}} \sqrt{\frac{d^{\alpha} K_{-1}}{2^{2 \alpha} K_{1}}},
$$

and the size of the final flocking can be estimated by

$$
\begin{gathered}
\max _{j \neq i}\left\|x_{i}(t)-x_{j}(t)\right\| \leq \sqrt{2 N}\left[\left(\sum_{i=1}^{N}\left\|x_{i 0}\right\|^{2}\right)^{(1 / 2)}\right. \\
\left.+\left(\sum_{i=1}^{N}\left\|v_{i 0}\right\|^{2}\right)^{(1 / 2)} T_{\max }\right] .
\end{gathered}
$$

Proof. Let $\quad x(t)=\left(x_{1}(t), x_{2}(t), \ldots, x_{N}(t)\right) \in \mathbb{R}^{d \times N}$ and $v(t)=\left(v_{1}(t), v_{2}(t), \ldots, v_{N}(t)\right) \in \mathbb{R}^{d \times N}$. Take the candidate Lyapunov functions:

$$
\begin{aligned}
& V(t):=\sum_{i=1}^{N}\left\|v_{i}(t)\right\|^{2}, \\
& X(t):=\sum_{i=1}^{N}\left\|x_{i}(t)\right\|^{2} .
\end{aligned}
$$

Then, a direct calculation from the definition of norm (15) and (21) yields that

$$
\begin{aligned}
& \sum_{i, j=1}^{N}\left\|v_{i}(t)-v_{j}(t)\right\|^{2}=2 N V(t), \\
& \sum_{i, j=1}^{N}\left\|x_{i}(t)-x_{j}(t)\right\|^{2}=2 N X(t) .
\end{aligned}
$$

It is easy to see from Definition 3 and (28) that function $V(t)$ tends to 0 in a fixed-time which means that the difference of all individuals' velocities will tend to zero in fixed time. In the following, we firstly show that $V(t)$ tends to zero in a fixed time. For $j \neq i$, a simple calculation from the derivative of $V(t)$ along the second equation of (13) gives that

$$
\begin{aligned}
\frac{\mathrm{d}}{\mathrm{d} t} V(t)= & 2 \sum_{i=1}^{N}\left\langle v_{i}(t), \frac{\mathrm{d}}{\mathrm{d} t} v_{i}(t)\right\rangle \\
= & \frac{2 K_{-1}}{N} \sum_{i, j=1}^{N} \psi\left(\left\|x_{i}-x_{j}\right\|\right)\left\langle v_{i}(t), \operatorname{sig}\left(v_{j}-v_{i}\right)^{1-2 \alpha}\right\rangle \\
& +\frac{2 K_{1}}{N} \sum_{i, j=1}^{N} \psi\left(\left\|x_{i}-x_{j}\right\|\right)\left\langle v_{i}(t), \operatorname{sig}\left(v_{j}-v_{i}\right)^{1+2 \alpha}\right\rangle .
\end{aligned}
$$

Then, a direct calculation yields

$$
\begin{aligned}
& \frac{2}{N} \sum_{i, j=1}^{N} \psi\left(\left\|x_{i}-x_{j}\right\|\right)\left\langle v_{i}(t), \operatorname{sig}\left(v_{j}-v_{i}\right)^{1-2 \alpha}\right\rangle \\
& =\frac{2}{N} \sum_{i, j=1}^{N} \psi\left(\left\|x_{i}-x_{j}\right\|\right)\left\langle v_{i}(t)-v_{j}(t), \operatorname{sig}\left(v_{j}-v_{i}\right)^{1-2 \alpha}\right\rangle \\
& \quad+\frac{2}{N} \sum_{i, j=1}^{N} \psi\left(\left\|x_{i}-x_{j}\right\|\right)\left\langle v_{j}(t), \operatorname{sig}\left(v_{j}-v_{i}\right)^{1-2 \alpha}\right\rangle \\
& =-\frac{2}{N} \sum_{i, j=1}^{N} \psi\left(\left\|x_{i}-x_{j}\right\|\right)\left\langle v_{j}(t)-v_{i}(t), \operatorname{sig}\left(v_{j}-v_{i}\right)^{1-2 \alpha}\right\rangle \\
& \quad-\frac{2}{N} \sum_{i, j=1}^{N} \psi\left(\left\|x_{i}-x_{j}\right\|\right)\left\langle v_{j}(t), \operatorname{sig}\left(v_{i}-v_{j}\right)^{1-2 \alpha}\right\rangle,
\end{aligned}
$$

which implies that

$$
\begin{aligned}
& \frac{2}{N} \sum_{i, j=1}^{N} \psi\left(\left\|x_{i}-x_{j}\right\|\right)\left\langle v_{i}(t), \operatorname{sig}\left(v_{j}-v_{i}\right)^{1-2 \alpha}\right\rangle \\
& -\frac{1}{N} \sum_{i, j=1}^{N} \psi\left(\left\|x_{i}-x_{j}\right\|\right)\left\langle v_{j}(t)-v_{i}(t), \operatorname{sig}\left(v_{j}-v_{i}\right)^{1-2 \alpha}\right\rangle \\
& -\frac{1}{N} \sum_{i, j=1}^{N} \psi\left(\left\|x_{i}-x_{j}\right\|\right) \sum_{k=1}^{\mathrm{d}}\left\|v_{j k}(t)-v_{i k}(t)\right\|^{2-2 \alpha} \cdot
\end{aligned}
$$

Employing Lemma 1 (i), one can easily obtain that

$$
\begin{aligned}
& \left(\sum_{k=1}^{d}\left\|v_{j k}(t)-v_{i k}(t)\right\|^{2-2 \alpha}\right)^{(1 /(2-2 \alpha))} \\
& \geq\left(\sum_{k=1}^{d}\left\|v_{j k}(t)-v_{i k}(t)\right\|^{2}\right)^{(1 / 2)}=\left\|v_{j}(t)-v_{i}(t)\right\|,
\end{aligned}
$$

which means

$$
\sum_{k=1}^{d}\left\|v_{j k}(t)-v_{i k}(t)\right\|^{2-2 \alpha} \geq\left\|v_{j}(t)-v_{i}(t)\right\|^{2-2 \alpha} .
$$

On the contrary, the same procedure of (31) may be easily adapted to obtain

$$
\begin{aligned}
& \frac{2}{N} \sum_{i, j=1}^{N} \psi\left(\left\|x_{i}-x_{j}\right\|\right)\left\langle v_{i}(t), \operatorname{sig}\left(v_{j}-v_{i}\right)^{1+2 \alpha}\right\rangle \\
& =-\frac{K_{1}}{N} \sum_{i, j=1}^{N} \psi\left(\left\|x_{i}-x_{j}\right\|\right) \sum_{k=1}^{d}\left\|v_{j k}(t)-v_{i k}(t)\right\|^{2+2 \alpha} .
\end{aligned}
$$

Then, it follows from Lemma 1 (i) that 


$$
\begin{aligned}
& \left(\sum_{k=1}^{d}\left\|v_{j k}(t)-v_{i k}(t)\right\|^{2+2 \alpha}\right)^{(1 /(2+2 \alpha))} \\
& \geq d^{1 / 2+2 \alpha-1 / 2}\left(\sum_{k=1}^{d}\left\|v_{j k}(t)-v_{i k}(t)\right\|^{2}\right)^{(1 / 2)} \\
& =d^{(1 /(2+2 \alpha))-(1 / 2)}\left\|v_{j}(t)-v_{i}(t)\right\|
\end{aligned}
$$

which implies that

$$
\left(\sum_{k=1}^{d}\left\|v_{j k}(t)-v_{i k}(t)\right\|^{2+2 \alpha}\right) \geq d^{-\alpha}\left\|v_{j}(t)-v_{i}(t)\right\|^{2+2 \alpha} \text {. }
$$

Note that $\inf _{s \geq 0} \psi(s) \geq \psi^{*}>0$; then, combining the abovementioned analysis gives that

$$
\begin{gathered}
\frac{\mathrm{d}}{\mathrm{d} t} V(t) \leq-\frac{\psi^{*} K_{-1}}{N} \sum_{i, j=1}^{N}\left\|v_{j}(t)-v_{i}(t)\right\|^{2-2 \alpha} \\
-\frac{\psi^{*} K_{1}}{N} d^{-\alpha} \sum_{i, j=1}^{N}\left\|v_{j}(t)-v_{i}(t)\right\|^{2+2 \alpha} .
\end{gathered}
$$

By Lemma 1 (ii) and (28), for $0<\alpha<(1 / 2)$, we have

$$
\begin{aligned}
& \sum_{i, j=1}^{N}\left\|v_{j}(t)-v_{i}(t)\right\|^{2-2 \alpha} \geq\left(\sum_{i, j=1}^{N}\left\|v_{j}(t)-v_{i}(t)\right\|^{2}\right)^{1-\alpha} \\
& \quad=(2 N)^{1-\alpha}[V(t)]^{1-\alpha} .
\end{aligned}
$$

From Lemma 2, for $0<\alpha<(1 / 2)$, we obtain

$$
\begin{aligned}
& \sum_{i, j=1}^{N}\left\|v_{j}(t)-v_{i}(t)\right\|^{2+2 \alpha} \geq N^{-2 \alpha}\left(\sum_{i, j=1}^{N}\left\|v_{j}(t)-v_{i}(t)\right\|^{2}\right)^{1+\alpha} \\
& \quad=2^{1+\alpha} N^{1-\alpha}[V(t)]^{1+\alpha} .
\end{aligned}
$$

For simplicity, we denote $V:=V(t)$. Then, combining the abovementioned inequalities with (37) yields that

$$
\frac{\mathrm{d}}{\mathrm{d} t} V \leq-\frac{\psi^{*} K_{-1}}{N}(2 N)^{1-\alpha} V^{1-\alpha}-\frac{\psi^{*} K_{1}}{N} \mathrm{~d}^{-\alpha} 2^{1+\alpha} N^{1-\alpha} V^{1+\alpha}
$$

which combining with Lemma 3 yields that

$$
V(t) \equiv 0, \quad \text { for } t \geq T_{\max },
$$

which means that

$$
v_{i}(t) \equiv v_{j}(t), \quad \text { for } t \geq T_{\max },
$$

where $T_{\max }$ is estimated by

$$
T_{\max }=\frac{2 N}{\alpha \psi^{*} K_{-1}(2 N)^{1-2 \alpha}} \sqrt{\frac{d^{\alpha} K_{-1}}{2^{2 \alpha} K_{1}}} .
$$

It follows from (40) that $V(t)$ is a nonincreasing function with respect to $t$, i.e.,

$$
V(0) \geq V(t), \quad \text { for } t \geq 0 .
$$

By a simple calculation from (27) and Cauchy-Schwartz inequality finds that

$$
\frac{\mathrm{d}}{\mathrm{d} t} X(t)=2 \sum_{i=1}^{N}\left\langle x_{i}(t), v_{i}(t)\right\rangle \leq 2 \sum_{i=1}^{N}\left\|x_{i}(t)\right\|\left\|v_{i}(t)\right\|
$$

$$
\leq 2 X^{(1 / 2)}(t) V^{(1 / 2)}(t) .
$$

Integrating the differential inequality (45) from 0 to $t$, we obtain

$$
X^{1 / 2}(t) \leq X^{1 / 2}(0)+\int_{0}^{t} V^{1 / 2}(s) \mathrm{d} s \quad \text { for } t \geq 0 .
$$

If $t \leq T_{\max }$, then it deduces from (44) that

$$
X^{1 / 2}(t) \leq X^{1 / 2}(0)+\int_{0}^{T_{\max }} V^{1 / 2}(s) \mathrm{d} s \leq X^{1 / 2}(0)+V^{1 / 2}(0) T_{\max }<\infty .
$$

If $t>T_{\max }$, then from (41), (44), and (46), we have

$$
\begin{aligned}
& X^{1 / 2}(t) \leq X^{1 / 2}(0)+\int_{0}^{T_{\max }} V^{1 / 2}(s) \mathrm{d} s+\int_{T_{\max }}^{t} V^{1 / 2}(s) \mathrm{d} s \\
& =X^{1 / 2}(0)+\int_{0}^{T_{\max }} V^{1 / 2}(s) \mathrm{d} s \\
& \leq X^{1 / 2}(0)+V^{1 / 2}(0) T_{\max } \leq C .
\end{aligned}
$$

Thus, combining the processing inequality with (28) implies that

$$
\begin{gathered}
\max _{i \neq j}\left\|x_{i}(t)-x_{j}(t)\right\| \leq \sqrt{2 N}\left(X^{1 / 2}(0)+V^{1 / 2}(0) T_{\max }\right) \\
=\sqrt{2 N}\left[\left(\sum_{i=1}^{N}\left\|x_{i 0}\right\|^{2}\right)^{1 / 2}+\left(\sum_{i=1}^{N}\left\|v_{i 0}\right\|^{2}\right)^{1 / 2} T_{\max }\right] .
\end{gathered}
$$

Therefore,

$$
\sup _{0 \leq t \leq \infty}\left\|x_{i}(t)-x_{j}(t)\right\|^{2} \leq C
$$

which combining with (42) indicates that all the conditions of Definition 3 are satisfied. Therefore, system (13) has a fixed-time flocking. The proof is completed.

Remark 3. Equation (49) means that the size of the final flocking can be estimated by the initial states of systems, the number of the particles, and the parameters of the systems. It also shows that the size of final flocking is proportional to the number of particles, which is in accordance with the fact.

Remark 4. Theorem 1 shows that the flocking can be established in a fixed-time which is independent of the initial states but merely of the parameters of the systems. This is 
quite different from Theorem 1 of [32] despite that we use the same communication function (11). Simultaneously, there is a big difference in Theorem 1 and the main results of [33], in which the author investigated the finite-time flocking.

Theorem 2. If all the conditions in Theorem 1 hold and suppose furthermore that the initial states of system (13) satisfy

$$
\min _{i \neq j}\left\|x_{i 0}-x_{j 0}\right\|>2\left\|v_{0}\right\| T_{\max },
$$

then system (13) has a noncollision fixed-time flocking, where $T_{\max }$ is defined in (25).

Proof. For $i \neq j$ and $i, j \in\{1,2, \ldots, N\}$, we make the following handy notations:

$$
\begin{aligned}
X_{i j}(t) & :=\left\|x_{i}(t)-x_{j}(t)\right\|, \\
V_{i j}(t) & :=\left\|v_{i}(t)-v_{j}(t)\right\| .
\end{aligned}
$$

Theorem 1 shows that system (13) has a fixed-time flocking. So, it is sufficient to prove that all particles do not collide during the process of flocking. That is, $X_{i j}(t)>0$, for all $t \geq 0$. For this, similar to (37), we have

$$
\frac{\mathrm{d}}{\mathrm{d} t}\|v(t)\|^{2} \leq-C_{1}\|v(t)\|^{2-2 \alpha}-C_{2}\|v(t)\|^{2+2 \alpha} .
$$

Furthermore,

$$
\frac{\mathrm{d}}{\mathrm{d} t}\|v(t)\| \leq-C_{1}\|v(t)\|^{1-2 \alpha}-C_{2}\|v(t)\|^{1+2 \alpha},
$$

which implies that

$$
\|v(t)\| \leq\left\|v_{0}\right\|
$$

where $C_{i}$ denotes the positive constants, whose value may vary from line to line. Hence, by the definition of $V_{i j}(t)$, one can easily obtain

$$
\left|V_{i j}(t)\right|=\left\|v_{i}(t)-v_{j}(t)\right\| \leq 2\|v\| \leq 2\left\|v_{0}\right\| .
$$

On the contrary, a standard calculation shows that

$$
\frac{\mathrm{d}}{\mathrm{d} t} X_{i j}^{2}(t)=2\left\langle x_{i}-x_{j}, v_{i}-v_{j}\right\rangle \leq 2\left\|x_{i}-x_{j}\right\|\left\|v_{i}-v_{j}\right\|=2 X_{i j} V_{i j} .
$$

Furthermore,

$$
\frac{\mathrm{d}}{\mathrm{d} t} X_{i j}(t) \leq V_{i j}
$$

Note that $V_{i j}=0$ for $t \geq T_{\max }$. So, combining (56) with (58) yields that

$$
\left|X_{i j}(t)-X_{i j}(0)\right|=\left|\int_{0}^{t} \frac{\mathrm{d} X_{i j}(s)}{\mathrm{d} s} \mathrm{~d} s\right| \leq \int_{0}^{T_{\max }}\left|V_{i j}(s)\right| \mathrm{d} s \leq 2\left\|v_{0}\right\| T_{\max } .
$$

Then, by the triangle inequality and (51), we obtain

$$
\left|X_{i j}(t)\right| \geq\left|X_{i j}(0)\right|-\left|X_{i j}(t)-X_{i j}(0)\right| \geq \min _{i \neq j}\left|X_{i j}(0)\right|-2\left\|v_{0}\right\| T_{\max }>0 .
$$

The proof is completed.

Remark 5. It is worth pointing out that to study the problem of collision for the particles, the usually method is to treat the particles as points without considering their actual sizes, for examples, see $[4,11,25,45]$. Therefore, the main contribution of Theorem 2 is providing a sufficient condition to guarantee the particles do not collide during the process of flocking and giving the rigorous mathematical arguments. However, it leads a gap that how about the particles with physical sizes, which will guide our further works.

\section{Simulation Examples}

To show the effectiveness of the theoretical analysis, three simulated examples are given in this part for illustration. Note that, in our model, $0<\alpha<(1 / 2)$ and $d \geq 1$ are required. So, for simplicity, we take $\psi^{*}=(1 / 2), \alpha=(1 / 4)$, and $d=1$ to simulate.

Example 1. We choose $N=4, K_{-1}=1$, and $K_{1}=2^{(1 / 2)}$. By (25), one can easily calculate that $T_{\max }=16.0000$. Moreover, the initial states are set by $x_{0}=[2,0,-2,-4]$ and $v_{0}=[1,2,3,4]$. Then, all the conditions of Theorem 2 are satisfied and the following simulation results (Figures 1-3) are obtained by Matlab.

From Figure 1, we see that the velocities of all particles are convergent to the same value after approximately $t=1$. Figure 2 indicates that the diameter of flocking occurs fluctuation before $t=1$. However, this fluctuation phenomenon vanishes after $t=1$ and the final diameter remains about 5.87. Meanwhile, Figure 3 shows that the least distance among all particles occurs fluctuation before $t=1$ and it is greater than zero. Moreover, the least distance maintains the value about 1.84 after $t=1$. Hence, Figure 3 shows up clearly that all particles do not collide all the time. Such fluctuation phenomenon reasonably clarifies the intrinsic characteristics of the flocking. Therefore, these simulation results effectively demonstrate that the system has a noncollision fixed-time flocking.

Example 2. Take $N=10, K_{-1}=1$, and $K_{1}=2^{(1 / 2)}$. From (25), we have $T_{\max }=25.2980$. The initial states are set by $x_{0}=[8,6,4,2,0,-2,-4,-6,-8,-10]$ and $v_{0}=[1,2,3,4,5$ $, 6,7,8,9,10]$. Then, all conditions of Theorem 2 are satisfied. By applying Matlab, we get the following simulation results (Figures 4-6).

It can be clearly seen from Figure 4 that the velocities of all particles tend to the convergent speed after about $t=0.5$. We can observe from Figure 5 that the diameter of the flocking fluctuates frequently before about $t=0.5$ and it verges to stability and remains about 17.98 after $t=0.5$. Meanwhile, Figure 6 also shows that the least distance among particles occurs fluctuation before about $t=0.5$. However, it does not vibrate anymore after $t=0.5$ and the 


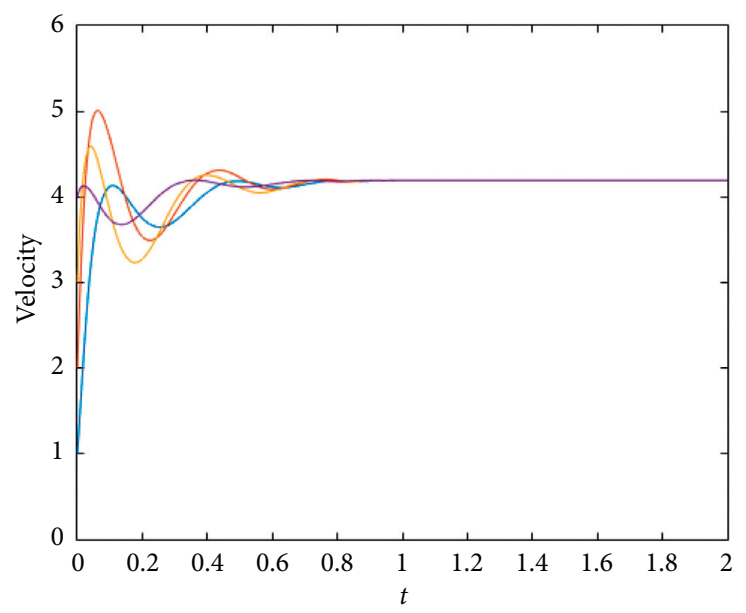

Figure 1: The velocities of particles.

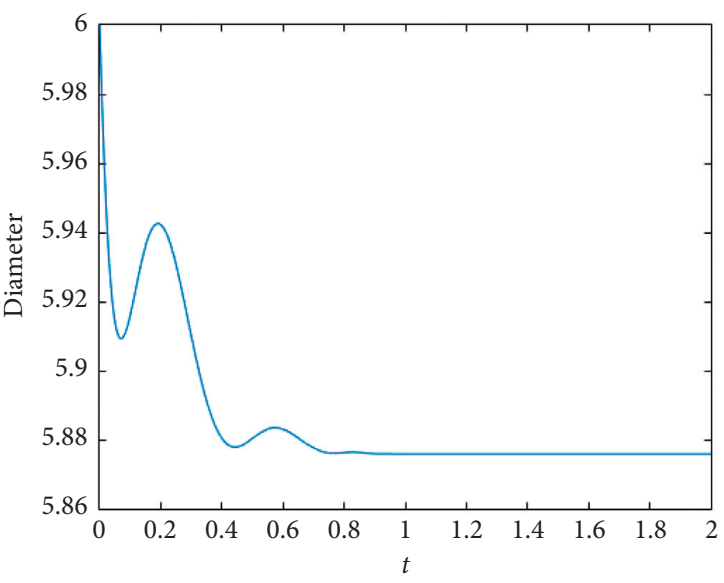

Figure 2: The diameter of flocking.

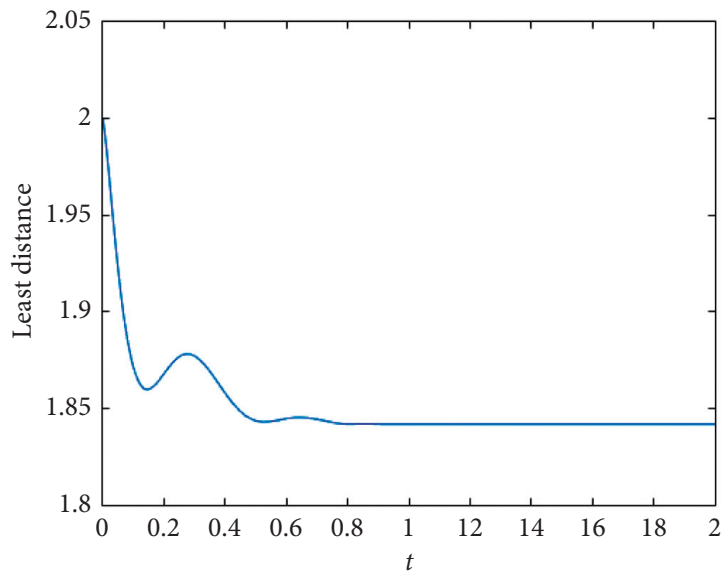

FIgure 3: The least distance among particles.

final value is about 1.973 , which means that any two agents do not collide. Therefore, these simulation results effectively demonstrate that the system has a noncollision fixed-time flocking.

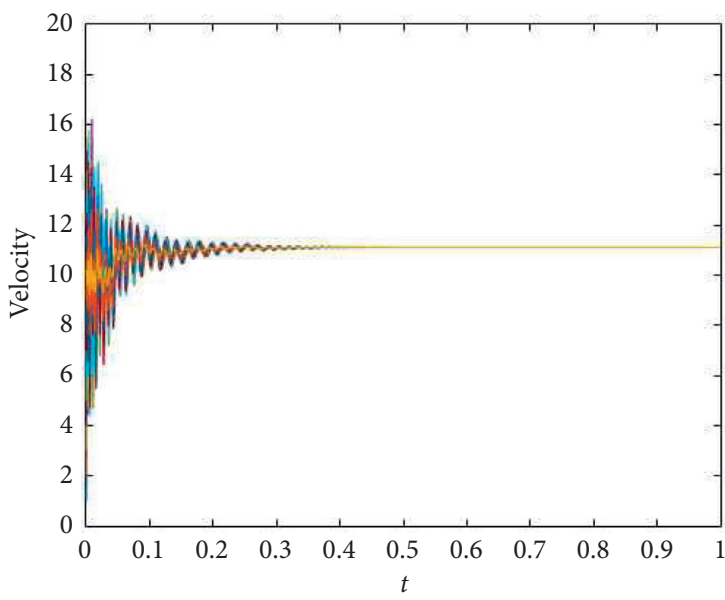

FIGURE 4: The velocities of particles.

To better illustrate the theoretical analysis of the main results, in the following Example 3, we add the number of particles to 25 , i.e., $N=25$, to simulate. In addition, the initial data in Example 3 are generated randomly since the initial data of Examples 1 and 2 are all set by man.

Example 3. Take $N=25, K_{-1}=1$, and $K_{1}=2^{(1 / 2)}$. The initial values of velocities and positions of all particles are generated randomly at the interval $(0,50)$ and $(-80,80)$, respectively. Then, all conditions of Theorem 2 are satisfied. Then, from (25), we have $T_{\max }=40.0000$, and by applying Matlab, we get the following simulation results (Figures 7-9).

Similar to the analysis of Examples 1 and 2, one can see that the velocities of all particles tend to the convergent speed after about $t=1.5$ in Figure 7. Figure 8 shows that the diameter of the flocking oscillates at high frequencies before about $t=0.1$, but it verges to stability and remains about 260 after $t=1.5$. This means that the size of the final flocking is about 260. At the same time, Figure 9 demonstrates clearly that the least distance among particles occurs fluctuation before about $t=0.1$. However, it does 


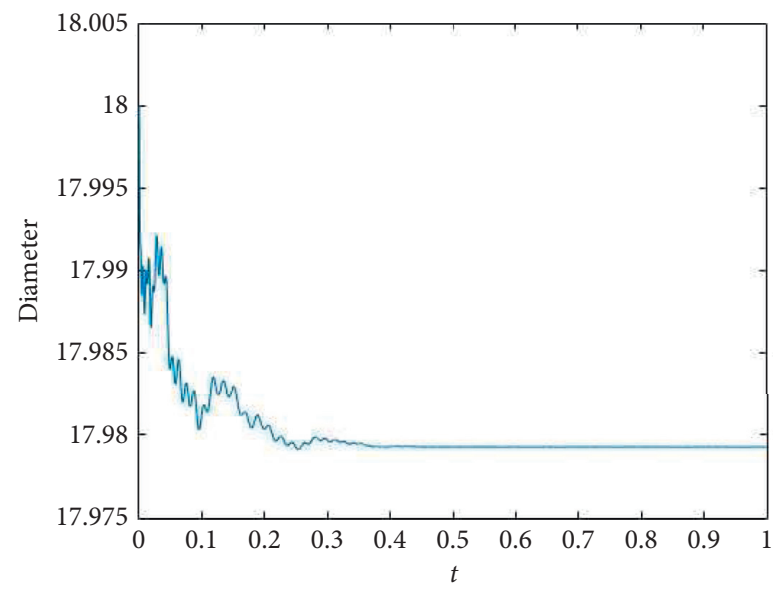

FIgUre 5: The diameter of flocking.

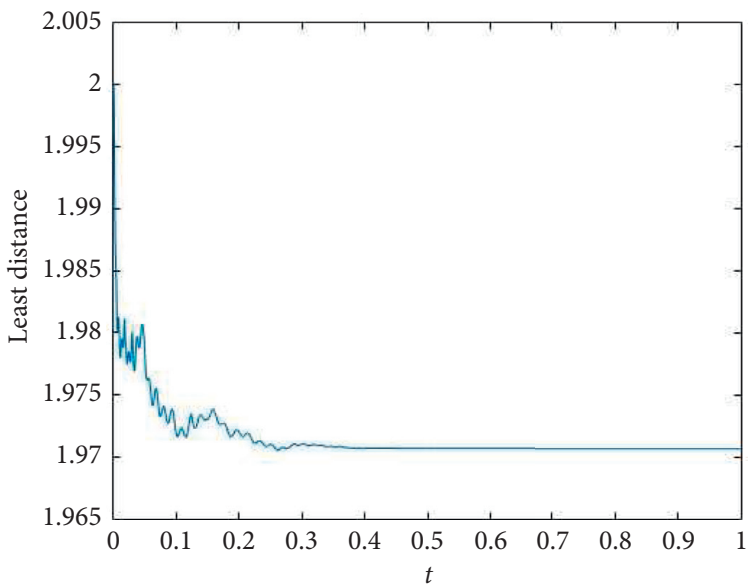

Figure 6: The least distance among particles.

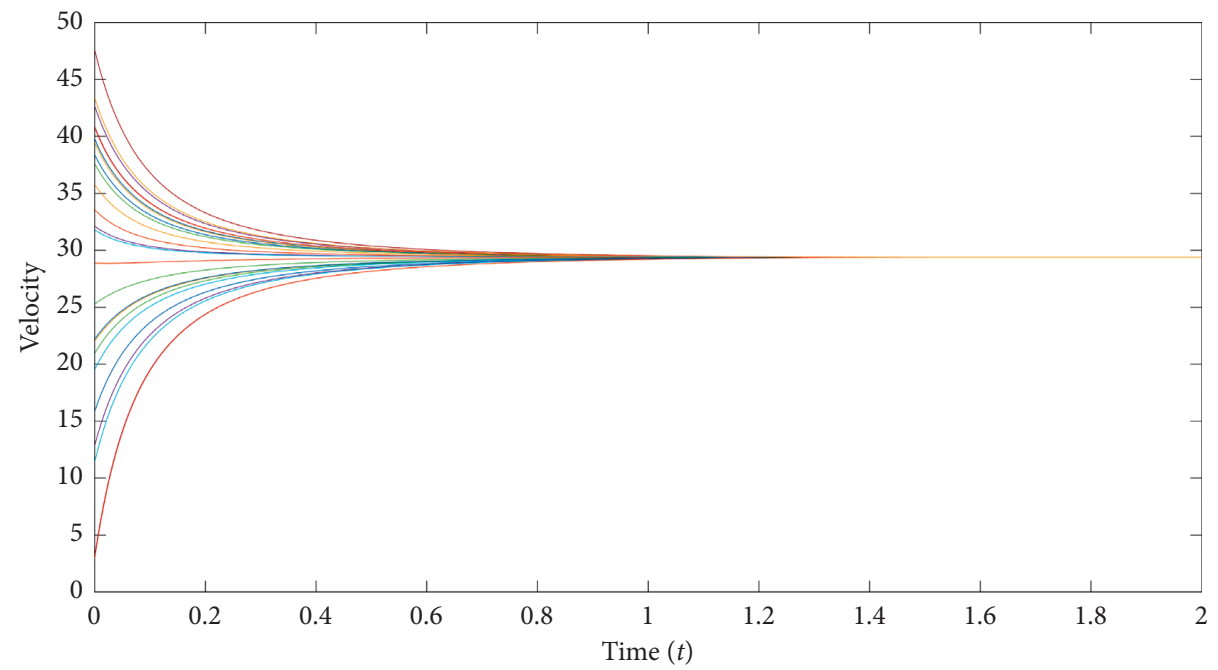

Figure 7: The velocities of particles. 


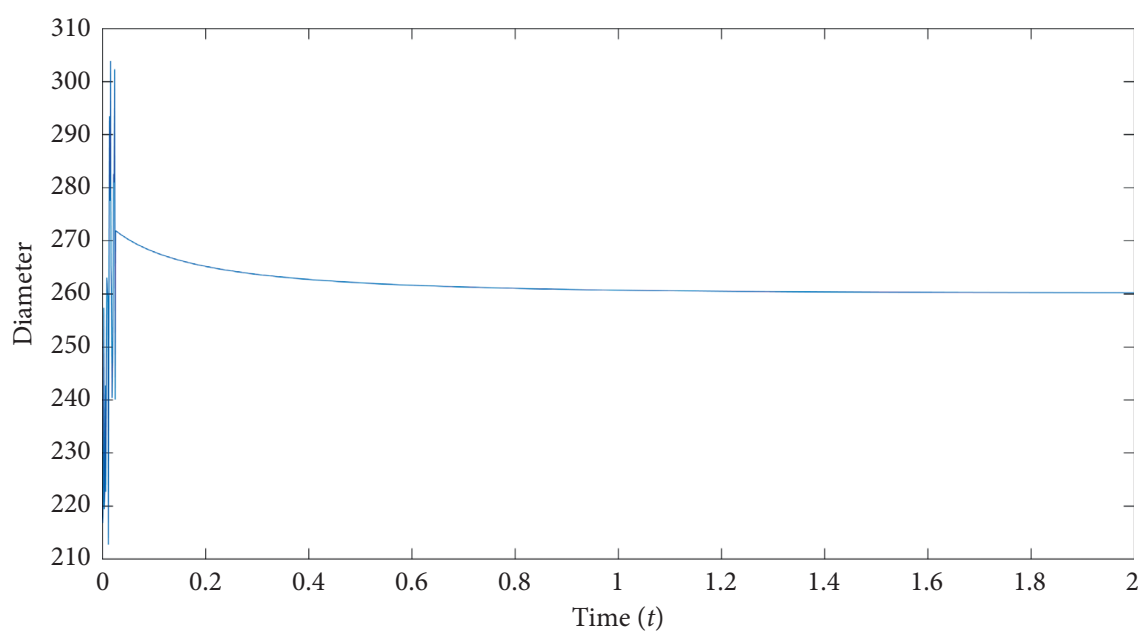

FIgURE 8: The diameter of flocking.

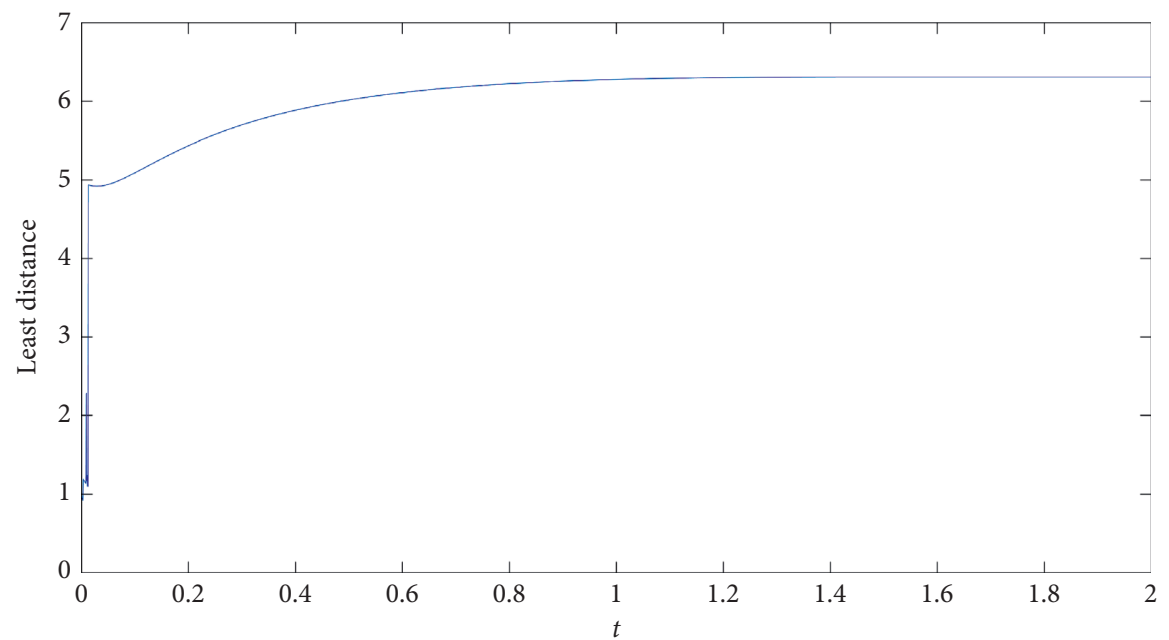

FIgURE 9: The least distance among particles.

not vibrate anymore after $t=1.5$ and the final value of it is about 6.32. No matter what, the least distance is greater than zero all the time, which means that any two particles do not collide. Therefore, these simulation results effectively illustrate that the system has a noncollision fixedtime flocking.

Additionally, we can see that the settling time in the abovementioned three examples is less than $T_{\max }$, respectively. The fluctuational phenomena of the least distance and the diameter of the systems reasonably clarify the intrinsic characteristics and the dynamic process of the flocking. Therefore, these simulation results sufficiently show that the flocking occurs in a fixed-time without collision and they effectively illustrate the theoretical analysis of Theorems 1 and 2 .

\section{Conclusion}

The fixed-time flocking with collision avoidance problem for the Cucker-Smale-type self-propelled particle model is investigated in this paper. By using a fixed-time stability theorem, the fixed-time flocking is received when the communication function has a positive infimum. Based on this result, a sufficient condition is given in the present paper to guarantee that any two particles do not collide during the process of the flocking. The theoretical analysis shows that the bound of the settling time does not depend on the initial states but merely on the design parameters. This distinctive feature ensures to reduce the calculation cost while achieving the agreement. Moreover, the diameter of the final flocking group can be estimated by the initial states and the number of the particles. It is worth emphasizing that the diameter of final flocking group is in proportion to the number of particles, which is in compliance with the fact. Finally, three numerical examples are provided to illustrate the effectiveness and validity of the theoretical results. Both the theoretical and numerical results provide a reasonable explanation for the birds that can flock in a short time. However, in this paper, we just consider the case that all the particles in the group exchange the information. In fact, the in-depth analytical work on the fixed-time flocking mechanism is still on the 
way, particularly the collision avoidance case. In the future, it will be important to focus on more detailed scenarios, such as the communication function with a switching protocol, time delays, or channel noises, to see whether the collision avoidance flocking takes place within a fixed time.

\section{Data Availability}

The data used to support the findings of this study are available from the corresponding author upon request.

\section{Conflicts of Interest}

The authors declare that they have no conflicts of interest.

\section{Acknowledgments}

This work was partially supported by the Hunan Natural Science Fund Youth Fund Project (no. 2018JJ3419) and Hunan Province Engineering Technology Research Center of Uranium Tailings Treatment Technology (no. 2018YKZX1008).

\section{References}

[1] A. Abdessameud and A. Tayebi, "Formation control of VTOL unmanned aerial vehicles with communication delays," Automatica, vol. 47, no. 11, pp. 2383-2394, 2011.

[2] W. Ren, "Distributed cooperative attitude synchronization and tracking for multiple rigid bodies," IEEE Transactions on Control Systems Technology, vol. 18, no. 2, pp. 383-392, 2010.

[3] G. Wen, W. Yu, Z. Li, X. Yu, and J. Cao, "Neuro-adaptive consensus tracking of multiagent systems with a high-dimensional leader," IEEE Transactions on Cybernetics, vol. 47, no. 7, pp. 1730-1742, 2017.

[4] J. A. Carrillo, M. Fornasier, J. Rosado, and G. Toscani, "Asymptotic flocking dynamics for the kinetic Cucker-Smale model," SIAM Journal on Mathematical Analysis, vol. 42, no. 1, pp. 218-236, 2010.

[5] Y. Liu and J. Wu, "Flocking and asymptotic velocity of the Cucker-Smale model with processing delay," Journal of Mathematical Analysis and Applications, vol. 415, no. 1, pp. 53-61, 2014.

[6] X. Li, Y. Liu, and J. Wu, "Flocking and pattern motion in a modified Cucker-Smale model," Bulletin of the Korean Mathematical Society, vol. 53, no. 5, pp. 1327-1339, 2016.

[7] H. Liu, X. Wang, Y. Liu, and X. Li, "On non-collision flocking and line-shaped spatial configuration for a modified singular Cucker-Smale model," Communications in Nonlinear Science and Numerical Simulation, vol. 75, pp. 280-301, 2019.

[8] J. A. Carrillo, A. Klar, S. Martin, and S. Tiwari, "Self-propelled interacting particle systems with roosting force," Mathematical Models and Methods in Applied Sciences, vol. 20, no. 1, pp. 1533-1552, 2010.

[9] J. Shen, "Cucker-Smale flocking under hierarchical leadership," SIAM Journal on Applied Mathematics, vol. 68, no. 3, pp. 694-719, 2007.

[10] S.-Y. Ha, K. Lee, and D. Levy, "Emergence of time-asymptotic flocking in a stochastic Cucker-Smale system," Communications in Mathematical Sciences, vol. 7, no. 2, pp. 453-469, 2009.
[11] F. Cucker and J.-G. Dong, "A general collision-avoiding flocking framework," IEEE Transactions on Automatic Control, vol. 56, no. 5, pp. 1124-1129, 2011.

[12] C. Pignotti and I. Reche Vallejo, "Flocking estimates for the Cucker-Smale model with time lag and hierarchical leadership," Journal of Mathematical Analysis and Applications, vol. 464, no. 2, pp. 1313-1332, 2018.

[13] S. M. Ahn, H. Choi, S.-Y. Ha, and H. Lee, "On collisionavoiding initial configurations to Cucker-Smale type flocking models," Communications in Mathematical Sciences, vol. 10, no. 2, pp. 625-643, 2012.

[14] Y.-P. Choi and Z. Li, "Emergent behavior of Cucker-Smale flocking particles with heterogeneous time delays," Applied Mathematics Letters, vol. 86, pp. 49-56, 2018.

[15] X. Wang, L. Wang, and J. Wu, "Impacts of time delay on flocking dynamics of a two-agent flock model," Communications in Nonlinear Science and Numerical Simulation, vol. 70, pp. 80-88, 2019.

[16] V. T. Haimo, "Finite time controllers," SIAM Journal on Control and Optimization, vol. 24, no. 4, pp. 760-770, 1986.

[17] S. P. Bhat and D. S. Bernstein, "Finite-time stability of continuous autonomous systems," SIAM Journal on Control and Optimization, vol. 38, no. 3, pp. 751-766, 2000.

[18] Y. Cao and W. Ren, "Finite-time consensus for multi-agent networks with unknown inherent nonlinear dynamics," Automatica, vol. 50, no. 10, pp. 2648-2656, 2014.

[19] B. Ning, J. Jin, J. Zheng, and Z. Man, "Finite-time and fixedtime leader-following consensus for multi-agent systems with discontinuous inherent dynamics," International Journal of Control, vol. 91, no. 6, pp. 1259-1270, 2018.

[20] G. Ji, C. Hu, J. Yu, and H. Jiang, "Finite-time and fixed-time synchronization of discontinuous complex networks: a unified control framework design," Journal of the Franklin Institute, vol. 355, no. 11, pp. 4665-4685, 2018.

[21] H. Wang, Z.-z. Han, Q.-y. Xie, and W. Zhang, "Finite-time synchronization of uncertain unified chaotic systems based on CLF," Nonlinear Analysis: Real World Applications, vol. 10, no. 5, pp. 2842-2849, 2009.

[22] J. Gortés, "Finite-time convergent gradient flows with applications to network consensus," Automatica, vol. 42, no. 11, pp. 1993-2000, 2006.

[23] L. Zhao and Y. Jia, "Finite-time consensus for second-order stochastic multi-agent systems with nonlinear dynamics," Applied Mathematics and Computation, vol. 270, pp. 278-290, 2015.

[24] M. Cai and Z. Xiang, "Adaptive neural finite-time control for a class of switched nonlinear systems," Neurocomputing, vol. 155, pp. 177-185, 2015.

[25] S. Li and X. Wang, "Finite-time consensus and collision avoidance control algorithms for multiple AUVs," Automatica, vol. 49, no. 11, pp. 3359-3367, 2013.

[26] X. Liu, M. Z. Q. Chen, H. Du, S. Yang, and S. Yang, "Further results on finite-time consensus of second-order multi-agent systems without velocity measurements," International Journal of Robust and Nonlinear Control, vol. 26, no. 14, pp. 3170-3185, 2016.

[27] Z. Zuo and L. Tie, "Distributed robust finite-time nonlinear consensus protocols for multi-agent systems," International Journal of Systems Science, vol. 47, no. 6, pp. 1366-1375, 2016.

[28] X. Liu, F. Alsaadi, W. Yu, and J. Cao, "Finite-time synchronisation control of complex networks via non-smooth analysis," IET Control Theory \& Applications, vol. 9, no. 8, pp. 1245-1253, 2015. 
[29] A. Khanzadeh and M. Pourgholi, "Fixed-time leader-follower consensus tracking of second-order multi-agent systems with bounded input uncertainties using non-singular terminal sliding mode technique," IET Control Theory \& Applications, vol. 12, no. 5, pp. 679-686, 2018.

[30] X. Liu, J. Cao, W. Yu, and Q. Song, "Nonsmooth finite-time synchronization of switched coupled neural networks," IEEE Transactions on Cybernetics, vol. 46, no. 10, pp. 2360-2371, 2016.

[31] X. Liu, J. Lam, W. Yu, and G. Chen, "Finite-time consensus of multiagent systems with a switching protocol," IEEE Transactions on Neural Networks and Learning Systems, vol. 27, no. 4, pp. 853-862, 2016.

[32] Y. Han, D. Zhao, and Y. Sun, "Finite-time flocking problem of a Cucker-Smale-type self-propelled particle model," Complexity, vol. 21, no. S1, pp. 354-361, 2006.

[33] J. G. Dong, "Finite-time flocking for multi-agent systems under long-range interactions," in Proceedings of the 2016 35th Chinese Control Conference (CCC), pp. 27-29, Chengdu, China, July 2016.

[34] V. Andrieu, L. Praly, and A. Astolfi, "Homogeneous approximation, recursive observer design, and output feedback," SIAM Journal on Control and Optimization, vol. 47, no. 4, pp. 1814-1850, 2008.

[35] A. Polyakov, "Nonlinear feedback design for fixed-time stabilization of linear control systems," IEEE Transactions on Automatic Control, vol. 57, no. 8, pp. 2106-2110, 2012.

[36] J. Fu and J. Wang, "Fixed-time coordinated tracking for second-order multi-agent systems with bounded input uncertainties," Systems \& Control Letters, vol. 93, pp. 1-12, 2016.

[37] Z. Zuo, "Nonsingular fixed-time consensus tracking for second-order multi-agent networks," Automatica, vol. 54, pp. 305-309, 2015.

[38] C. Hu, J. Yu, Z. Chen, H. Jiang, and T. Huang, "Fixed-time stability of dynamical systems and fixed-time synchronization of coupled discontinuous neural networks," Neural Networks, vol. 89, pp. 74-83, 2017.

[39] Q. Xiao, H. Liu, X. Wang, and Y. Huang, "A note on the fixedtime bipartite flocking for nonlinear multi-agent systems," Applied Mathematics Letters, vol. 99, Article ID 105973, 2020.

[40] M. Defoort, K. Veluvolu, M. Djemai, A. Polyakov, and G. Demesure, "Leader-follower fixed-time consensus for multi-agent systems with unknown non-linear inherent dynamics," IET Control Theory \& Applications, vol. 9, no. 14, pp. 2165-2170, 2015.

[41] Y. Zhang, Y. Yang, Y. Zhao, and G. Wen, "Distributed finitetime tracking control for nonlinear multi-agent systems subject to external disturbances," International Journal of Control, vol. 86, no. 1, pp. 29-40, 2013.

[42] A. Filippov and F. Arscott, Differential Equations with Discontinuous Righthand Sides, Springer, Berlin, Germany, 1988.

[43] G. Hardy, J. Littlewood, and G. Polya, Inequalities, Cambridge University Press, Cambridge, UK, 1952.

[44] F. Cucker and S. Smale, "Emergent behavior in flocks," IEEE Transactions on Automatic Control, vol. 52, no. 5, pp. 852862, 2007.

[45] Z. Z.-H. Guan, F. F.-L. Sun, Y. Y.-W. Wang, and T. Li, "Finitetime consensus for leader-following second-order multi-agent networks," IEEE Transactions on Circuits and Systems, vol. 59, no. 11, pp. 2646-2654, 2012. 